\title{
A measuring complex for control the uniformity of the light output of scintillators
}

\author{
S.O.Tretyak, O.V.Popkova \\ Institute for Scintillation Materials, STC "Institute for Single Crystals", \\ National Academy of Science of Ukraine, \\ 60 Nauki Ave., 61001 Kharkiv, Ukraine
}

Received January 19, 2018

\begin{abstract}
A complex was developed and manufactured for monitoring the uniformity of the light output of ZnSe scintillators when excited by X-ray radiation, suitable for servicing mass production of scintillation elements and investigating the distribution of light output over the surface of scintillators.
\end{abstract}

Keywords: measuring complex, scintillator, light output, X-ray radiation.

\begin{abstract}
Разработана и изготовлена установка для контроля равномерности световыхода $\mathrm{ZnSe}$ сцинтилляторов при возбуждении рентгеновским излучением, пригодная для обслуживания массового производства сцинтилляционных элементов и исследования распределения световыхода по поверхности сцинтилляторов.
\end{abstract}

Вимірювальний комплекс для контролю рівномірності світлового виходу сцинтиляторів. С.О.Третьяк, О.В.Попкова

Розроблено та виготовлено установку для контролю рівномірності світловиходу ZnSe сцинтиляторів при збудженні рентгенівським випромінюванням, придатна для обслуговування масового виробництва сцинтиляційних елементів і дослідження розподілу світловиходу вздовж поверхні сцинтиляторів.

While manufacturing the detectors of ionizing radiation based on the scintillation effect, there is a need to study the light output of the scintillators used. To measure the scintillation characteristics of $\mathrm{ZnSe}(\mathrm{Te})$ in the radiation instrumentation department, a complex was developed for measuring the uniformity of the light output of scintillators, oriented both at mass production as well at research.

During the development of the complex, a scheme was used to investigate the object under study for lumen, i.e. the source of the exciting radiation and the measuring photodetector were located on opposite sides of the investigated sample (investigation into the lumen), which repeats the scheme for constructing the inspection introscopes (Fig. 1) [1, 2]. In contrast to the scheme used in the stand [3], the research object (scintillator) moves relative to the stationary source of radiation and the photodetector in the developed complex.

The displacement system provided the possibility of two-coordinate travel on the field $200 \times 200 \mathrm{~mm}^{2}$ with a resolution of $0.1 \mathrm{~mm}$.

The source of radiation in the measurements was the X-ray apparatus RAAP-150M with a perforated anode. The energy of the X-ray beam during operation was $90 \ldots 140 \mathrm{kV}$. For localizing the irradiation zone, lead collimators were installed. The diameter of the output part of the collimator varied from 1 to $4 \mathrm{~mm}$, depending on the geometry of the samples being studied.

The photodetector was a silicon photodiode with $5 \times 5 \mathrm{~mm}^{2}$ measurement cross-sec- 


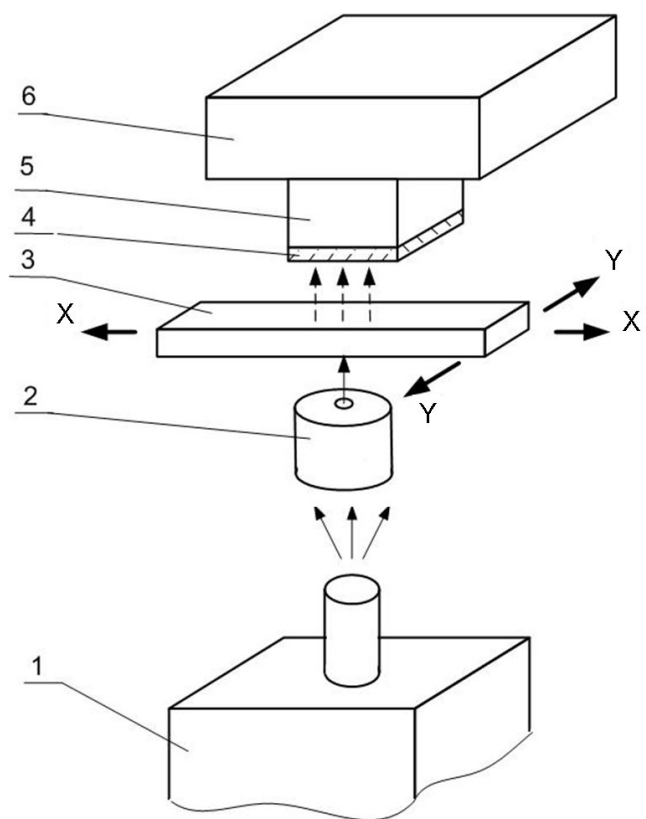

Fig. 1. Stand measuring the relative light output of scintillators. 1 - X-ray tube; $2-$ Collimator; 3 - Object of measurement (scintillator); 4 - Protective filter; 5 - Photodetector; 6 - Amplifier.

tion. To protect the photodiode of the receiver from direct exposure to an X-ray beam, a stopping filter of a GSO crystal was used absorbing nearly all incident X-rays, while the proper scintillation luminescence of the filter crystal is located in the blue region of the spectrum [4] that does not affect the silicon photodiode.

Further, the analog signal was amplified by a current-voltage instrument amplifier (Fig. 1) and fed to a 12-bit ADC board built into the computer.

The computer also controlled the movement mechanism of the cassette with crystals relative to the $\mathrm{X}$-ray source. The discreteness of displacement along both axes is $0.1 \mathrm{~mm}$, and the dimensions of the working zone are $200 \times 200 \mathrm{~mm}^{2}$.

The stand does not allow measuring the light output as a physical quantity, since it is not calibrated and not verified. The relative light output is measured in comparison to the selected standard. For each individual task it is necessary to select its distinct standard. In order to eliminate the influence of the geometry of the experimental samples and the standard on the measurement results, the standards were chosen as closely as possible in the same shape and size with working scintillators.

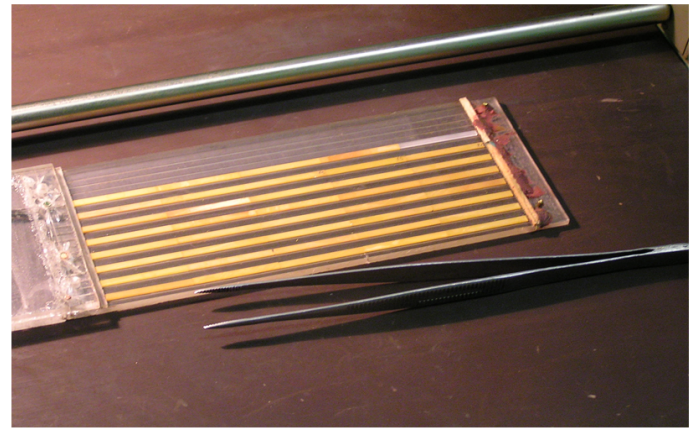

Fig. 2. Cassette with scintillation crystals $\mathrm{ZnSe}(\mathrm{Te}) 25.4 \times 2.3 \times 0.6 \mathrm{~mm}^{3}$.

So at this stand, a technique for controlling scintillators for detectors of inspection introscopes was worked out.

The scintillation crystal for this problem is a parallelepiped of $\mathrm{ZnSe}$ measuring $25 \times 2.3 \times 0.6 \mathrm{~mm}^{3}$ (or $18.8 \times 2.3 \times 0.6 \mathrm{~mm}^{3}$ ).

Preliminarily, the scintillator control was carried out according to the measurement technique, which allows controlling the light output at three points along the length of the crystal and having a carousel for stacking samples at 30 positions or at the firm's bench (AMS-1"Smith Heimann"). All measurement operations were manual, which limited the performance of the operation to 100 pieces/shift, and manual fixation of measurement results could introduce additional errors, reducing the reliability of the results.

To localize the irradiation zone, a lead collimator with a hole diameter of $3 \mathrm{~mm}$ was installed. Since the diameter of the collimated beam is greater than the width of the sample, the data on the homogeneity of the light output of the scintillator are investigated only along the axis of the crystal. To remove this characteristic, each sample was measured at five points with a uniform pitch of $5.1 \mathrm{~mm}$.

While developing a new technique for increasing productivity, a group load method was used with automatic measurement itself. The control is carried out by all $100 \%$ of the products transferred to the measurements with the issuance of a passport protocol that displays each product individually.

The algorithm of the measurement program included measuring the parameters of the light output of the reference crystal ZnSe (in sizes completely analogous to the working samples) that was located at the first position of the cassette, and then all other working samples. 


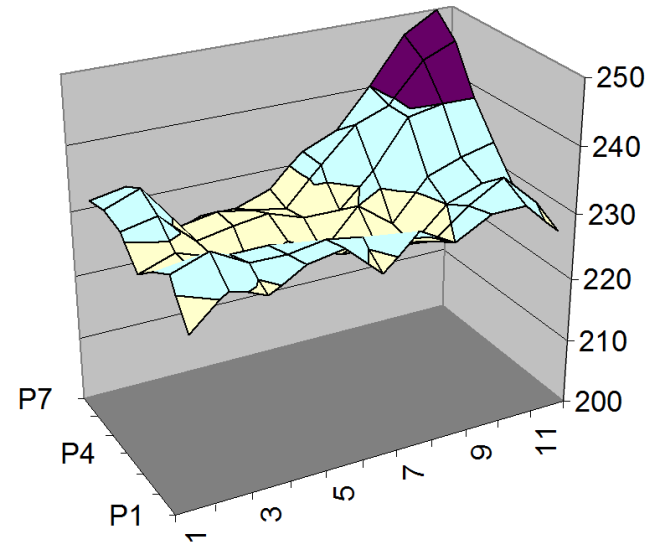

Fig. 3. The distribution diagram of the light output on the plate $\mathrm{ZnSe}(\mathrm{Te}) 24 \times 10 \times 1 \mathrm{~mm}^{3}$.

For these scintillators, a cassette was used, which allows placing 50 samples. The samples were stacked in a cassette in ten rows of five pieces in a row. The appearance of the cassette with samples is shown in Fig. 2. The distance between the rows is $5 \mathrm{~mm}$, which was the minimum possible value with this collimator. Moving the cassette was done in a shuttle way - this allowed to reduce the processing time of each load. The average value for each sample while processing the measurement results was calculated, as well as the largest deviations in "+" and "-" were determined. All the values after normalization on the sample crystal and sorting by the size of the light output were recorded in the final protocol. Additionally, monotonicity parameters of each sample could be output in the protocol. On the basis of these protocols, samples were sorted and the shipping batches of scintillators were formed.

Manual operations left only the placement of samples in the measuring cassette, their return to the shipping container and the initial setting of the parameters of the measurement program. In general, the measurement site is now able to measure up to 1000 scintillators per shift.

This stand also allows you to scan the surface of the scintillation screen to investigate the distribution of the light output characteristics. For example, Fig. 3 shows the distribution of the light output along the surface of a ZnSe (Te) screen with the size of $32 \times 24 \mathrm{~mm}^{2}$. The scanning step was
$2 \mathrm{~mm}$ in both coordinates; the diameter of the collimator was $2 \mathrm{~mm}$.

Such a study makes it possible to control the uniformity of light output over the sample and to analyze the shape of the resulting surface in order to look for technological solutions for improving the uniformity, which is important when creating screens for X-ray and medical devices. In addition, scanning the surface of the scintillator is used to control the workpieces for detectors. These blanks are in the form of plates of rectangular or round shape measuring $20 \ldots 50 \mathrm{~mm}$ and thickness from $2.5 \mathrm{~mm}$ to $6-7 \mathrm{~mm}$. The reference plate for $\mathrm{ZnSe}(\mathrm{Te})$ is $2 \mathrm{~mm}$ thick, which is scanned simultaneously with the sample in one session. The obtained results are then normalized to the value of the light output of the reference crystal with a size of $24 \times 10 \times 1 \mathrm{~mm}^{3}$ (the average light output of the standard is conventionally assumed to be $100 \%$ ) and is issued in the form of a two-dimensional diagram showing the light output of the sample in the form of a surface.

This method of processing proved to be convenient also for controlling blanks, selecting the required for further processing, determining the orientation of the subsequent cutting of round blanks, and the like.

\section{Conclusions}

The device was developed and fabricated, and the technique of measuring the relative light output of ZnSe crystals suitable for servicing mass production of scintillation elements and investigating the distribution of light output over the surface of scintillators was developed.

Methods for studying the light output of scintillators of various geometric shapes have been employed.

\section{References}

1. The X-ray Introscope System Poliskan-4, Prospect, stri@isc.kharkov.com.

2. Heimann, Prospects, Germany (2003), http://www.heimannsystems.com.

3. Yu.P.Korneev, A.N.Krinitsyn, V.I.Kryshkin and et al., The Scintillation Counters Control Stand, IHEP Preprint 2003-31, Protvino (2003).

4. Yu.A.Tsirlin, M.E.Globus, E.P.Sysoeva, Optimization of Detection of Gamma Radiation by Scintillation Crystals, Energoatomizdat, Moscow (1991) [in Russian]. 